\title{
An Algorithm for Computing the Restriction Scaffold Assignment Problem in Computational Biology
}

\author{
Justin Colannino
}

\author{
School of Computer Science \\ McGill University \\ Montréal, Québec, Canada
}

\author{
Godfried Toussaint
}

Technical Report SOCS-TR-2005.2, January 2005.

\begin{abstract}
Let $S$ and $T$ be two finite sets of points on the real line with $|S|+|T|=n$ and $|S|>|T|$. The restriction scaffold assignment problem in computational biology assigns each point of $S$ to a point of $T$ such that the sum of all the assignment costs is minimized, with the constraint that every element of $T$ must be assigned at least one element of $S$. The cost of assigning an element $s_{i}$ of $S$ to an element $t_{j}$ of $T$ is $\left|s_{i}-t_{j}\right|$, i.e., the distance between $s_{i}$ and $t_{j}$. In 2003 Ben-Dor, Karp, Schwikowski and Shamir [2] published an $O(n \log n)$ time algorithm for this problem. Here we provide a counter-example to their algorithm and present a new algorithm that runs in $O\left(n^{2}\right)$ time, improving the best previous complexity of $O\left(n^{3}\right)$.
\end{abstract}

\section{Introduction}

In the context of measuring the similarity of musical rhythms with the goal of performing a phylogenetic analysis of rhythms, Toussaint [8] proposed the use of the swap-distance. A comparison of this distance measure with other rhythm dissimilarity measures shows it to be superior in several respects [9]. In [8] and [9] the rhythms are represented as binary sequences, where a ' 1 ' denotes the onset of a note and a ' 0 ' denotes a silence. Furthermore, all the rhythms compared have the same length, $n$ bits, with the same number $k$ of 1's (or onsets). A swap operation on a string consists of interchanging two adjacent elements in that string. The swap distance between two binary strings is defined as the minimum number of swap operations needed to transform one string into the other. In this restricted version of the problem, computing the swap-distance is very simple. For all $i$, the $i$ th ' 1 ' of one string must move to the position of the $i$ th ' 1 ' of the second string. Therefore the number of swaps needed for one such operation is the difference between their indices (which may be viewed as integer $x$-coordinates). The swap distance is the sum of all the $k$ differences, which may be trivially computed in $O(n)$ time given the two binary sequences as input. Note that actually performing the swaps may require $\Omega\left(n^{2}\right)$ swaps.

In a more general setting, the two rhythms have different values of $k$, and the algorithm described in the preceding will not work. In order to capture the attributes of the swap distance measure on two binary strings $S$ and $T$ where $S$ has more elements than $T$, Toussaint proposed the directed swap distance, which was first applied to the phylogenetic analysis of Flamenco rhythms by Dìaz-Báñez et al. [4]. The directed swap distance is defined as the minimum number of swaps required to move every element of $S$ to the index of an element of $T$, with the restriction that every element of $T$ must have at least one element of $S$ moved to its index.

The directed swap distance may be viewed as a version of the linear assignment problem [6], where the cost of an assignment between an element $i$ of $S$ and an element $j$ of $T$ is the distance between $i$ and $j$. 


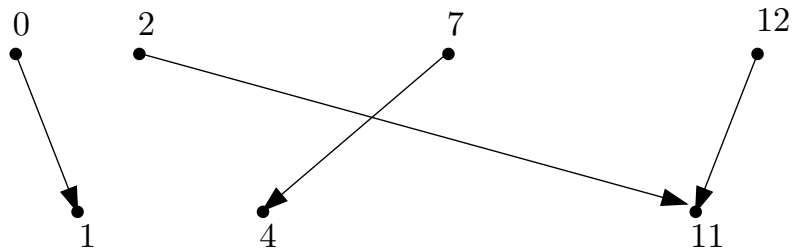

(a)

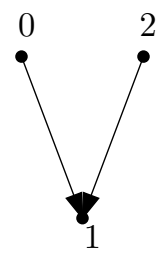

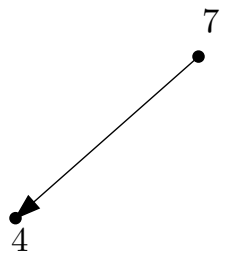

(b)

Figure 1: (a) A surjection between the two one dimensional sets $S=\{0,2,7,12\}$ and $T=\{1,4,11\}$. (b) A minimal surjection between $S$ and $T$.

Furthermore, we may consider the more general input consisting of two sets of numbers on the real line rather than binary sequences. Here the real numbers play the role of the indices of the 1's in the binary sequence. In this setting, if both sets have equal cardinalities, the simple algorithm described in the preceding for binary sequences may still be used after sorting the sets, thus yielding an $O(n \log n)$ time algorithm.

An alternate way of viewing the directed swap distance is as a surjection, $\psi$, between two sets of elements $S$ (the source) and $T$ (the target) on the interval $(0, X)$ where $|S| \geq|T|$. This mapping is bound by the constraint that each element of $T$ must have at least one element of $S$ mapped to it. More formally the directed swap distance may be expressed as a surjection as follows:

$$
\min _{\psi} \sum_{s \in S}|s-\psi(s)| .
$$

Any surjection that satisfies the preceding equation we call a minimal surjection. Figure 1 depicts two different surjections between two sets of points on the line, one of which is minimal. Note that all the points actually have zero $y$-coordinates; they are shown in this way merely for the purpose of clarity.

In 1979 the philosopher Graham Oddie proposed using surjections to measure the distance between two theories expressed in a logical language [7]. In 1997 Eiter and Mannila extended this idea by expressing theories as models, and thus as points in a metric space [5]. This gave them a new distance measure in a metric space which they called the surjection distance. The surjection distance between two sets $S$ and $T$ is defined as follows:

$$
\min _{\psi} \sum_{s \in S} \delta(s, \psi(s)),
$$

where $\delta$ is a distance metric on the space, and $\psi$ is a surjection between $S$ and $T$. They also proposed an algorithm for computing the surjection distance in $O\left(n^{3}\right)$ time, where $n=|S|$, by reducing the problem to finding a minimum-weight perfect matching in an appropriate graph.

In 2003, Ben-Dor et al. [2], in the context of the shotgun sequencing problem in computational biology, introduced a modified assignment problem similar to the directed swap problem where the points are real numbers on the line rather than bits in a binary string: the restriction scaffold assignment problem. They also presented an $O(n \log n)$ algorithm to compute this assignment problem. Their result relies heavily on a result of Karp and Li [6] which provides a linear time algorithm (after sorting) for computing the one-toone assignment problem in the special case where all the points lie on a line. In the one-to-one assignment problem between $S$ and $T$ some elements of $S$ remain unassigned.

In this note we give a counter-example to the algorithm of Ben-Dor et al., [2] for computing the restriction scaffold assignment problem, and show that the problem may be solved in $O\left(n^{2}\right)$ time. 


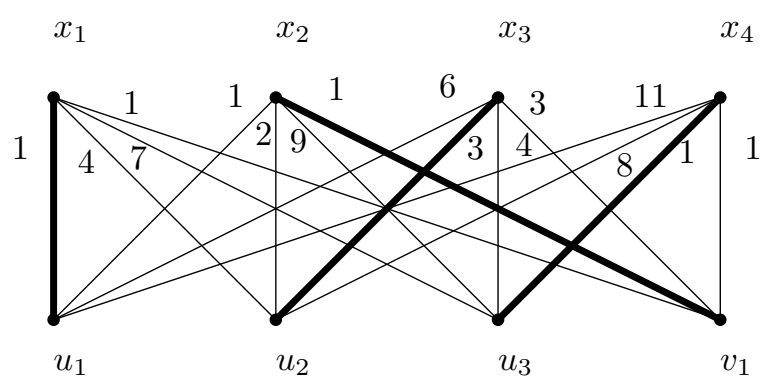

Figure 2: The bipartite graph created from $S=\{0,2,7,12\}, T=\{1,4,11\}$. The bold lines represent the minimum perfect bipartite matching, which has a cost of 6 .

\section{The Algorithm of Eiter and Mannila}

Since our $O\left(n^{2}\right)$ algorithm is inspired by, and based on, some results of Eiter and Mannila [5], we describe in this section their main results for the sake of clarity and completion. The proofs of these results may be found in [5]. Their algorithm is based on a reduction to finding a minimum weight perfect matching in a suitable bipartite graph. The reduction makes use of the following observation.

Lemma 2.1. Let $\psi$ be a minimal surjection from $S$ to $T$. Then for any $s_{i} \neq s_{j}$ if $\psi\left(s_{i}\right)=\psi\left(s_{j}\right)$ the distance from $s_{i}$ to $\psi\left(s_{i}\right)$ is not more than the distance from $s_{i}$ to any other element of $T$.

Taking advantage of this result their algorithm creates $k=|S|-|T|$ auxiliary vertices. These vertices serve as dummy nodes, for which the distance to any $s \in S$ is made equal to that of the shortest distance from $s$ to any element of $T$. This allows the graph to effectively simulate the situation where two or more vertices are mapped to the same element of $T$.

This construction is carried out as follows. For two sets, $S$ and $T$ in a metric space, create a complete bipartite weighted graph $G=(X \cup Y, E, w)$. For each $s_{i} \in S$ construct a vertex $x_{i} \in X$. For each $t_{j} \in T$ create a vertex $u_{j} \in U$. Finally, for $k=|S|-|T|$, create a set of nodes $V=\left\{v_{1}, \ldots ., v_{k}\right\}$, and let $Y=(U \cup V)$. The weight function $w$ is defined as follows:

$$
w(e)= \begin{cases}e=\left(x_{i}, u_{j}\right), & \delta\left(s_{i}, t_{j}\right) \\ e=\left(x_{i}, v_{j}\right), & \min _{t \in T} \delta\left(s_{i}, t\right)\end{cases}
$$

where $s_{i}, t_{j}$ are the set elements corresponding to the vertices $x_{i}, u_{j}$, respectively.

Figure 2 illustrates this construction with the sets $S=\{0,2,7,12\}$ and $T=\{1,4,11\}$. The bold lines represent the minimum perfect bipartite matching, and correspond to the minimal surjection depicted in Figure 1 (b). Let $w(M)$ denote the minimum-weight perfect matching of the resulting graph, and let $c(\psi)$ denote the cost of the minimum surjection distance between $S$ and $T$. Eiter and Mannila also prove the following theorem.

Theorem 2.2. $c(\psi)=w(M)$.

Letting $n$ equal to $|S|$, Eiter and Mannila note that this reduction yields an $O\left(n^{3}\right)$ time algorithm since the construction takes $O\left(n^{2}\right)$ time and their matching algorithm of choice takes $O\left(n^{3}\right)$ time for a complete graph [3].

\section{A Counter-Example to the Algorithm of Ben-Dor et al.}

Ben-Dor et al. [2] presented an $O(n \log n)$ time algorithm for computing the restriction scaffold assignment problem. In this section we describe their algorithm and exhibit an example on which the algorithm does not 


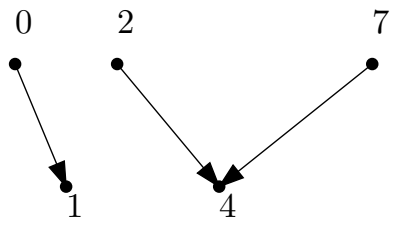

(a)

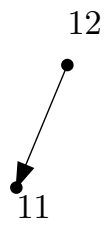

12

11

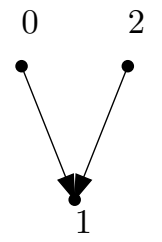

Figure 3: (a) The assignment returned by the algorithm in [2]. (b) A minimal assignment.

yield the claimed optimal solution.

The key idea in their algorithm is to split the problem into two parts. First, the minimal one-to-one assignment, $F$, between $S$ and $T$ is found, leaving out a set $E \subset S$. Then each element of $E$ is mapped to its nearest neighbor in $S$. The attractive feature of this procedure is that it runs in linear time provided that it uses the Karp-Li algorithm to solve the first part of the problem (the one-to-one assignment) on points that are already sorted.

A simple counter-example to this algorithm follows. Consider the two sets $S=\{0,2,7,12\}$ and $T=$ $\{1,4,11\}$ shown in Figure 3. The minimum one-to-one assignment function, $F$, is

$$
\begin{aligned}
& F(0)=1 \\
& F(2)=4 \\
& F(12)=11
\end{aligned}
$$

which has a total cost of 4 . Adding in the distance between 7 and its nearest neighbor in $T$, 4 , gives a total cost of $4+3=7$. However, this is not a minimum-cost surjective assignment. We have already seen a solution for these two sets in Section 2, where, using the algorithm of Eiter and Mannila, we were able to find a surjection $\psi$ between $S$ and $T$ with values,

$$
\begin{aligned}
& \psi(0)=1 \\
& \psi(2)=1 \\
& \psi(7)=4 \\
& \psi(12)=11
\end{aligned}
$$

which has a total cost of 6 , thus providing a counter-example to the algorithm. Figure 3 illustrates this counter-example.

\section{The New $O\left(n^{2}\right)$ Algorithm}

In this section we propose a new $O\left(n^{2}\right)$ time algorithm for computing the the restriction scaffold assignment problem (as well as the directed swap distance). The algorithm is based on a reduction to the problem of computing the single source shortest path problem in a weighted directed acyclic graph. The construction is inspired by the graph-theoretic approach of Eiter and Mannila, and relies heavily on the following lemma, sometimes called the quadrangle inequality [1].

Lemma 4.1. Let $S$ and $T$ be sets of points on the line. Also, let a distance function $\delta(s, t)=|s-t|$. Then for $a, b \in S$ where $a<b$, and $c, d \in T$ where $c<d$,

$$
\delta(a, c)+\delta(b, d) \leq \delta(a, d)+\delta(b, c)
$$

Proof. Three cases arise as pictured in Figure 4.

Case $1: a$ and $b$ are both less than $c$ or, symmetrically, greater than $d$ 


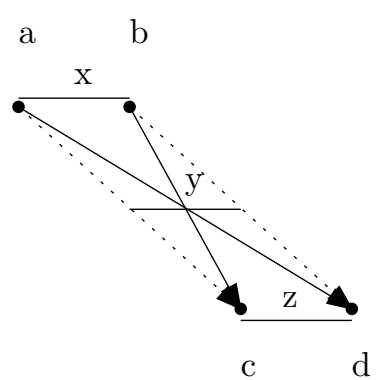

case 1

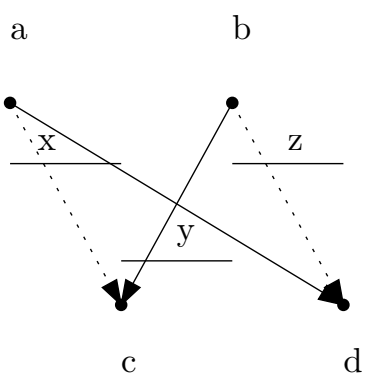

case 2

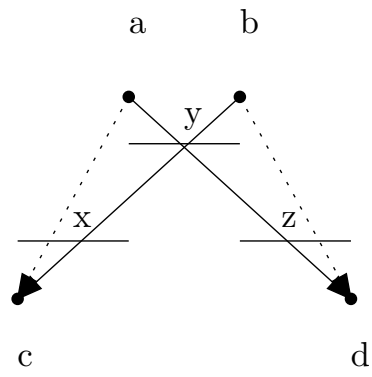

case 3

Figure 4: Illustration of lemma 4.1. The dashed lines represent the smaller distance.

Let $x=|a-b|, y=|b-c|$ and $z=|c-d|$. Then we have $\delta(a, c)+\delta(b, d)=x+2 y+z=$ $\delta(a, d)+\delta(b, c)$.

Case 2: Either a or b, but not both, are in between $c$ and $d$

Let $x=|a-c|, y=|c-b|$ and $z=|b-d|$. Then, $\delta(a, c)+\delta(b, d)=x+z \leq x+2 y+z=$ $\delta(a, d)+\delta(b, c)$.

Case $3:$ Both $a$ and $b$ are in between $c$ and $d$

Let $x=|c-a|, y=|a-b|$ and $z=|b-d|$. Then, $\delta(a, c)+\delta(b, d)=x+z \leq x+2 y+z=$ $\delta(a, d)+\delta(b, c)$

We relate this lemma to the directed swap distance with the following corollary.

Corollary 4.2. Let $S$ and $T$ be sets of points on the line. Then there exists a minimal surjection $\psi^{*}$ from $S$ to $T$ such that for all $s_{i}<s_{j}, \psi^{*}\left(s_{i}\right) \leq \psi^{*}\left(s_{j}\right)$.

Proof. Let $\psi$ be a minimal surjection function that does not satisfy the lemma. Then there must exist some $s_{i}<s_{j}$ where $\psi\left(s_{j}\right)<\psi\left(s_{i}\right)$. Let $\psi\left(s_{j}\right)=t_{k}$ and $\psi\left(s_{i}\right)=t_{l}$. Consider a new function, $\psi^{*}$, which differs from $\psi$ only by having $\psi^{*}\left(s_{i}\right)=t_{k}$ and $\psi^{*}\left(s_{j}\right)=t_{l}$. By the quadrangle inequality lemma we have that $\sum_{s \in S}\left|s-\psi^{*}(s)\right| \leq \sum_{s \in S}|s-\psi(s)|$, thus proving our hypothesis.

The first thing to note is that since all elements of $S$ and $T$ lie on a line, corollary 4.2 implies that no crossings can occur in an optimal assignment. Therefore, we know that the first element of $S$ must be mapped to the first element of $T$. Furthermore, also by corollary 4.2, given that $s_{i}$ is mapped to $t_{j}$, we can predict that $s_{i+1}$ is optimally mapped to either $t_{j}$ or $t_{j+1}$. The proposed algorithm builds a directed acyclic graph whose structure takes advantage of these two observations.

Let $S, T$ be sets of integers on the interval $(0, X)$, where $|S| \geq|T|$, and let $s_{i}$ be the $i$ th element of $S$. We construct a weighted directed acyclic graph $G=(V, E)$ in the following way. For each pair $i, j$ where $1 \leq i \leq|T|$ and $i \leq j \leq(i+|S|-|T|)$, we create a vertex, $v_{i, j}$. From each $v_{i, j}$ we add an edge to $v_{i, j+1}$ with weight $\left|t_{i}-s_{j+1}\right|$, and an edge to $v_{i+1, j+1}$ with weight $\left|t_{i+1}-s_{j+1}\right|$, provided $v_{i, j+1}$ and $v_{i+1, j+1}$ exist. Finally, we create a node labeled 'start' and put one edge from 'start' to $v_{1,1}$ with weight $\left|s_{1}-t_{1}\right|$. An example of this construction is shown in Figure 5. The bold lines represent the minimum-length path through the graph, and 


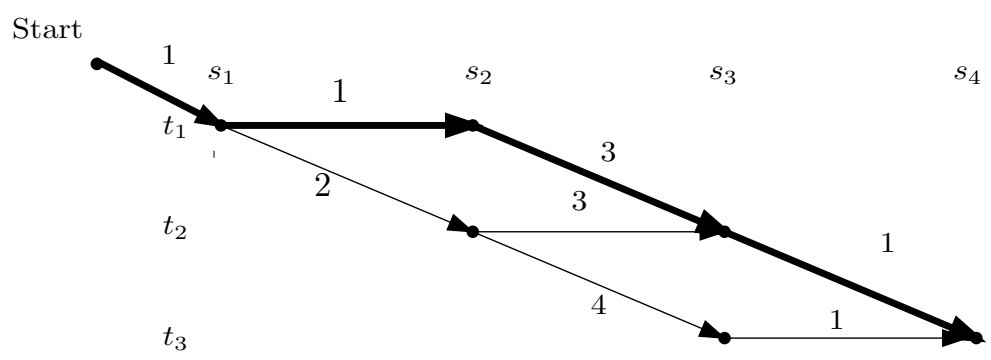

Figure 5: A directed acyclic graph created from $S=\{0,2,7,12\}, T=\{1,4,11\}$. The bold lines represent the shortest path through the graph, which has weight 6 .

correspond to the minimum surjection shown in Figure 1 (b).

It remains to show that the weight of the minimum-length path $w(P)$ returned from 'start' to $v_{|T|,|S|}$ is equal to the cost of the minimum surjection between $S$ and $T, c(\psi)$. We do this by showing first that $c(\psi) \leq w(P)$, and then that $c(\psi) \geq w(P)$.

Lemma 4.3. $c(\psi) \leq w(P)$.

Proof. From a minimum weight path $P^{*}$ we create a surjection $\psi$ in the following way. For each vertex, $v_{i, j}$ that $P^{*}$ passes through, let $\psi\left(s_{j}\right)=t_{i}$. We can verify that $\psi$ is indeed a surjection by noting two things. First, each $s_{j}$ is mapped to only one $t_{i}$, since edges from $v_{i, j}$ to $v_{i+1, j}$ do not exist in the construction. Second, every $t_{i}$ receives at least one $s_{j}$, since no path exists from $v_{i, x}$ to $v_{i+2, y}$ without first passing through some $v_{i+1, z}$. This surjection $\psi$ has a cost equal to that of $P^{*}$, since for $j=2$ to $|S|,\left|s_{j}-\psi\left(s_{j}\right)\right|=w\left(v_{x, j-1}, v_{y, j}\right)$ and $\left|s_{1}-\psi\left(s_{1}\right)\right|=w\left(\right.$ start, $\left.v_{1,1}\right)$. Hence, $c(\psi) \leq w(P)$.

Lemma 4.4. $c\left(\psi^{\prime}\right) \geq w(P)$ :

Proof. From an optimal surjection $\psi^{\prime}$ we can create a path $P$ using the following method. By corollary 4.2 , for $j=1$ to $|S|-1$, there are only two possibilities. When $\psi\left(s_{j}\right)=t_{i}$ and $\psi\left(s_{j}+1\right)=t_{i}$ add the edge, $\left(v_{i, j}, v_{i, j+1}\right)$ to $P$. Otherwise, $\psi\left(s_{j}\right)=t_{i}$ and $\psi\left(s_{j}+1\right)=t_{i}+1$, so we add the edge $\left(v_{i, j}, v_{i+1, j+1}\right)$ to P. Finally we add the edge ('start', $v_{1,1}$ ).

It follows that $P$ is a path through the constructed graph from 'start' to $v_{|S|+|T|}$. A consequence of lemma 4.2 is that $\psi^{\prime}\left(s_{j}\right)=t_{i}$ implies that $\psi^{\prime}\left(s_{j+1}\right)=t_{i}$ or $\psi^{\prime}\left(s_{j+1}\right)=t_{i+1}$, which means that the corresponding path $P$ is connected in $G$. Also, notice that there are exactly $k=|S|-|T|$ integers, $j$, where $\psi^{\prime}\left(s_{j}\right)=t_{i}$ and $\psi^{\prime}\left(s_{j+1}\right)=t_{i}$. Thus, after $|S|$ steps along $P$ we must be at node $v_{|S|,|S|-k}=v_{|S|,|T|}$. From the definition of the weight function it follows that $P$ has a weight equal to that of $\psi^{\prime}$. Hence, $P$ is a valid path and $c\left(\psi^{\prime}\right) \geq w(P)$.

Theorem 4.5. $c(\psi)=w(P)$.

Proof. The result is immediate from lemmas 4.3 and 4.4.

As for the complexity of this method, first let $|S|=n$ and $|T|=m$. Since in our construction each vertex has at most two edges pointing to it, we have that $|E|=O(|V|)$. Also note that $|V|=n *(n-m)$. Therefore the construction takes $O(|V|+|E|)=O(|V|)$ time, and the single source shortest path algorithm for directed acyclic graphs also takes $O(|V|+|E|)=O(|V|)$ time [3]. Therefore the entire algorithm runs in $O(|V|)$ time, which is $O\left(n^{2}\right)$ in the worst case, thus improving on the previous best complexity of $O\left(n^{3}\right)$ for this problem due to Eiter and Mannila [5]. 


\section{Acknowledgement}

The authors thank Marc Berndl and Paco Gomez for helpful discussions concerning this research.

\section{References}

[1] Alok Aggarwal, Amotz Bar-Noy, Samir Khuller, Dina Kravets, and Baruch Schieber. Efficient minimum cost matching and transportation using the quadrangle inequality. Journal of Algorithms, 19(1):116-143, 1995.

[2] Amir Ben-Dor, Richard M. Karp, Benno Schwikowski, and Ron Shamir. The restriction scaffold problem. Journal of Computational Biology, 10(2):385-398, 2003.

[3] Thomas H. Cormen, Charles E. Leiserson, Ronald L. Rivest, and Clifford Stein. Introduction to Algorithms. MIT Press, Cambridge, Mass., 2001.

[4] J. Miguel Dìaz-Báñez, Giovanna Farigu, Francisco Gomez, David Rappaport, and Godfried Toussaint. El compás flamenco: A phylogenetic analysis. In Proceedings of BRIDGES: Mathematical Connections in Art, Music, and Science, pages 61-70, July 30 to August 12004.

[5] Thomas Eiter and Heikki Mannila. Distance measures for point sets and their computation. Acta Informatica, 34(2):109-133, 1997.

[6] Richard M. Karp and Shuo-Yen R. Li. Two special cases of the assignment problem. Discrete Mathematics, 13(2):129 - 142, 1975.

[7] G. Oddie. Likeness to Truth. D. Reidel Publishing Company, Dordrecht, Holland, 1986.

[8] Godfried T. Toussaint. Classification and phylogenetic analysis of African ternary rhythm timelines. In Proceedings of BRIDGES: Mathematical Connections in Art, Music and Science, pages 25-36, Granada, Spain, July 23-27 2003.

[9] Godfried T. Toussaint. A comparison of rhythmic similarity measures. In Proc. 5th International Conference on Music Information Retrieval, pages 242-245, Barcelona, Spain, October 10-14 2004. Universitat Pompeu Fabra. 